\title{
Objectively measured sedentary behavior and physical activity in a sample of Finnish adults: a cross-sectional study
}

Pauliina Husu ${ }^{1 *}$, Jaana Suni ${ }^{1}$, Henri Vähä-Ypyä ${ }^{1}$, Harri Sievänen ${ }^{1}$, Kari Tokola ${ }^{1}$, Heli Valkeinen ${ }^{2}$, Tomi Mäki-Opas ${ }^{3}$ and Tommi Vasankari ${ }^{1}$

\begin{abstract}
Background: Regular physical activity (PA) confers many positive effects on health and well-being. Sedentary behavior (SB), in turn, is a risk factor for health, regardless of the level of moderate to vigorous PA. The present study describes the levels of objectively measured SB, breaks in SB, standing still and PA among Finnish adults.

Methods: This cross-sectional analysis is based on the sub-sample of the population-based Health 2011 Study of Finnish adults. The study population consisted of 18-to-85-year old men and women who wore a waist-worn triaxial accelerometer (Hookie AM 20) for at least 4 days, for at least $10 \mathrm{~h}$ per day $(n=1587)$ during a week. PA and SB were objectively assessed from the raw accelerometric data using novel processing and analysis algorithms with mean amplitude deviation as the processing method. The data was statistically analyzed using cross-tabulations, analysis of variance and analysis of covariance.

Results: The participants were on average 52 years old, $57 \%$ being women. Participants were sedentary $59 \%$ of their waking wear time, mainly sitting. They spent $17 \%$ of the time standing still, $15 \%$ in light intensity PA, $9 \%$ in moderate PA and less than $1 \%$ in vigorous PA. Participants aged 30-39 years had the highest number of breaks in SB per day. Younger participants ( $<30$ years of age) had more moderate and vigorous PA than older ones ( $\geq 60$ years of age), and 30-60-year-olds had the greatest amount of light PA.

Conclusions: Participants spent nearly $60 \%$ of their waking time sedentary, and the majority of their daily PA was light. From a public health perspective it is important to find effective ways to decrease SB as well as to increase the level of PA. Our analysis method of raw accelerometer data may allow more precise assessment of dose-response relationships between objectively measured PA and SB and various indicators of health and well-being.
\end{abstract}

Keywords: Physical activity, Sedentary behavior, Accelerometer, Adults

Abbreviations: $\mathrm{Cl}$, Confidence interval; HEPA, Health-enhancing physical activity; MET, Metabolic equivalent; MVPA, Moderate-to-vigorous physical activity; PA, Physical activity; SB, Sedentary behavior; SD, Standard deviation

\section{Background}

Physical activity (PA) confers many positive effects on health and well-being [1]. Regular PA can be a safe and low-cost medicine for several health problems, and importantly, be an effective means to prevent these problems and related disability. Sedentary behavior $(\mathrm{SB})$ is a distinct behavior from moderate to vigorous PA (MVPA) [2]. By definition, SB means any waking behavior

\footnotetext{
* Correspondence: pauliina.husu@uta.fi

${ }^{1}$ The UKK Institute for Health Promotion Research, Tampere, Finland

Full list of author information is available at the end of the article
}

characterized by an energy expenditure $\leq 1.5$ METs in a sitting or reclining posture [3]. Thus, SB is a separate construct from physical inactivity, which indicates low levels of PA, not reaching the level of the current recommendation for health-enhancing PA [3]. SB covers several facets, for example purpose, environment, type, posture, social and time, all of which include several sub-categories, for example purpose can cover work, education, transport, eating, rest and leisure [4]. Several studies have indicated that SB is a risk factor for cardiorespiratory and metabolic health [5-8], musculoskeletal 
health [9], depression [10] and mortality [1, 11], regardless of the level of MVPA [12], but not all studies confirm this independency [13].

Measurement of both SB and PA is important for describing the prevalence of these behaviors in different populations, in determining secular trends in these behaviors, for evaluating effects of interventions and for determining dose-response influence on specific health outcomes [14]. Traditionally the knowledge on SB and PA has largely rested on self-reports [15] whose validity and reliability is quite poor [16-19].

Technological development has enabled measuring new aspects of PA and SB [20, 21]. New devices have made it possible to record volume, duration, intensity and frequency of activities [22]. These new tools also offer a possibility to categorize individuals more specifically according to their PA and SB levels, which may be useful in targeting health promotion actions more precisely. For example, accelerometer data allows identification of individual PA patterns which may help to personalize PA counseling and goal setting However, criterion validity of these objective measurements varies a lot [23]. Most population studies describing objectively measured PA and SB have used count-based methods using varying analysis algorithms [24-29], which makes the direct comparison between studies very challenging. Thus, tri-axial accelerometer storing information as raw data instead of proprietary units (counts) has been proposed the method of choice when accurate and specific assessment of SB and PA is of primary importance $[21,30,31]$. Utilization of raw tri-axial acceleration data may advance comparisons between studies and different devices.

The purpose of the present study was to describe the levels of SB and PA in a sample of Finnish adults using raw data from tri-axial accelerometer which were analyzed with novel, validated analysis algorithms [30, 32]. More specifically, the study aimed at describing the amount of SB, number of breaks in SB, amount of standing still and the amount and intensity (light, moderate, vigorous) of PA. Furthermore, a novel classification scheme for different PA levels and number of steps is proposed. Combining several accelerometer parameters may help identifying individual activity patterns and thus facilitate targeted actions to reduce SB and promote PA more effectively.

\section{Methods}

\section{Participants}

The study is a part of a population-based Health 2011 Study [33], which is a multifactorial health examination study conducted with a stratified two-stage cluster sample of Finnish adults. Mainland Finland was divided into 20 strata defined by the 15 largest towns and the remaining rural areas based on the five university hospital regions. The sample represents the target population of each stratum, with exception of immigration after year 2000 . The present study is based on the sub-sample $(n=4916)$ of the Health 2011 Study, of which $50 \%(n=2455)$ participated in the study. The data collection of the study was conducted between August 2011 and March 2012. Of those 2055 (84 \%) were willing to take the waist-worn accelerometer (Hookie AM 20, Traxmeet Ltd, Espoo, Finland) for seven-day-measurements. The present study sample comprised of 18-85-year-old men and women $(n=2040)$ who used the accelerometer to sufficient extent; at least 4 days with a minimum of $10 \mathrm{~h}$ per day. Previous studies (e.g. [27, 34]) have used the similar criteria. In the present study 1587 persons met this criterion. All participants gave a signed informed consent before participation.

\section{Measurement of PA and SB}

The accelerometer was attached to a flexible belt on the right hip and the participants were instructed to wear the belt for seven consecutive days during waking hours, except during showering and other water activities. The accelerometers were returned by mail to the research institute where the stored data were copied to a hard disk and analyzed later. The Hookie AM 20 device measures and stores the acceleration of the device in three orthogonal $\mathrm{x}, \mathrm{y}$ and $\mathrm{z}$ directions at sampling rate of $100 \mathrm{~Hz}$. The resultant acceleration (i.e. the magnitude of the acceleration vector) was determined from these three components. Then the mean amplitude deviation (MAD) of the resultant was analyzed in $6 \mathrm{~s}$ epoch length [30]. The MAD value describes the mean value of the dynamic acceleration component and it is independent of static gravity component. PA was categorised into three intensity categories based on metabolic equivalents (MET): light, moderate and vigorous. Classification has been validated with simultaneous measurements of MAD values and oxygen consumption (mean $r=0.958$ ) during a non-stop pace-conducted walking and running test on an indoor track [32]. Light PA was defined as activity corresponding 1.5-2.9 MET, moderate activity as 3.0-5.9 MET and vigorous activity more than 6 MET [35]. Intensities of cycling and Nordic skiing were slightly underestimated by the accelerometer used and water-based activities were excluded.

Steps were identified by applying the method described by Ying et al. [36] and Mizell [37]. Instead of adaptive thresholds a fixed threshold was used. According to the definition of SB [3], time spent in sitting and reclining positions were combined to indicate $\mathrm{SB}$, while standing still was analysed separately. It is possible to accurately determine whether the participant is standing, sitting or lying by applying the tri-axial information from 
the accelerometer. Since the body position during walking is upright and the direction of Earth's gravity vector is constant, the vertical position (angle) of the accelerometer can be identified during normal walking. This known position can then be used for recognizing different body postures. In standardized conditions, standing can be separated from sitting or lying with $100 \%$ accuracy, and sitting from lying with $95 \%$ accuracy [38]. Daily amount of stand-ups (breaks in sedentary time) was calculated on the basis of the number of lying/sitting periods ending with a standing up. The standing up was detected if the MAD value was greater than $50 \mathrm{mg}$ (milligravity) for the preceding or same epoch when the measured posture changed to standing. Participants whose daily measurement time was over $20 \mathrm{~h}$ were considered to have slept with the accelerometer. To avoid possible bias in SB time, their waking wear time was limited to $20 \mathrm{~h}$ and the exceeding time was reduced from the lying time.

\section{PA classification}

The classification scheme of PA was formed on the basis of intensity and duration of PA in addition to the number of daily steps (Fig. 1). The classification was based on the $1 \mathrm{~min}$ moving average (mean of 10 last $6 \mathrm{~s}$ epochs) of the resultant acceleration. The participants meeting the current health-enhancing PA (HEPA) recommendation for aerobic activity (at least $150 \mathrm{~min}$ of moderate intensity or at least 75 min of vigorous intensity aerobic PA per week consisting of bouts lasting at least $10 \mathrm{~min}$ at the time or an equivalent combination of both intensities) [35] formed the HEPA-group. The HEPA-group was further divided in two sub-groups according to the number of mean daily steps. The step limits were based on the distribution of the present study sample utilizing the limits proposed by Tudor-Locke et al.
[39]. Those taking less than 12000 steps per day formed HEPA-low group and those taking 12000 steps or more were named HEPA-high group. The participants not meeting the HEPA recommendation, but taking on average more than 5000 steps per day formed PA-group. This group was further broken down to three sub-groups: PA-low taking 5000-7499 steps per day, PA-mid taking 7500-9999 steps and PA-high taking 10000 or more steps per day. The participants taking on average less than 5000 steps per day [40] were regarded as inactive.

\section{Assessment of background characteristics}

In order to describe the background characteristics of the present study sample the data collected by a Health 2011 Study -questionnaire was utilized. The participants were asked to report their educational background (no vocational education, vocational education, university degree) and marital status (cohabited, single). They also were asked to assess, how they perceived their health status and physical fitness (good, fairly good, poor), as well as the level of their PA lasting at least $10 \mathrm{~min}$ at the time separately for aerobic and musculoskeletal activities. This PA data was used to assess whether the participants met the current recommendation for HEPA [35]: 1) met the whole recommendation (moderate intensity aerobic PA for at least 150 min per week, vigorous intensity PA for at least $75 \mathrm{~min}$ per week or an equal amount of MVPA spread throughout the week and musculoskeletal activity at least twice a week), 2) met aerobic part, 3) met musculoskeletal part and 4) did not meet the recommendation.

\section{Statistical analysis}

The data was analyzed by SPSS software, version 22 (SPSS Inc, Chicago IL) using descriptive methods (crosstabulation, analysis of variance, analysis of covariance).

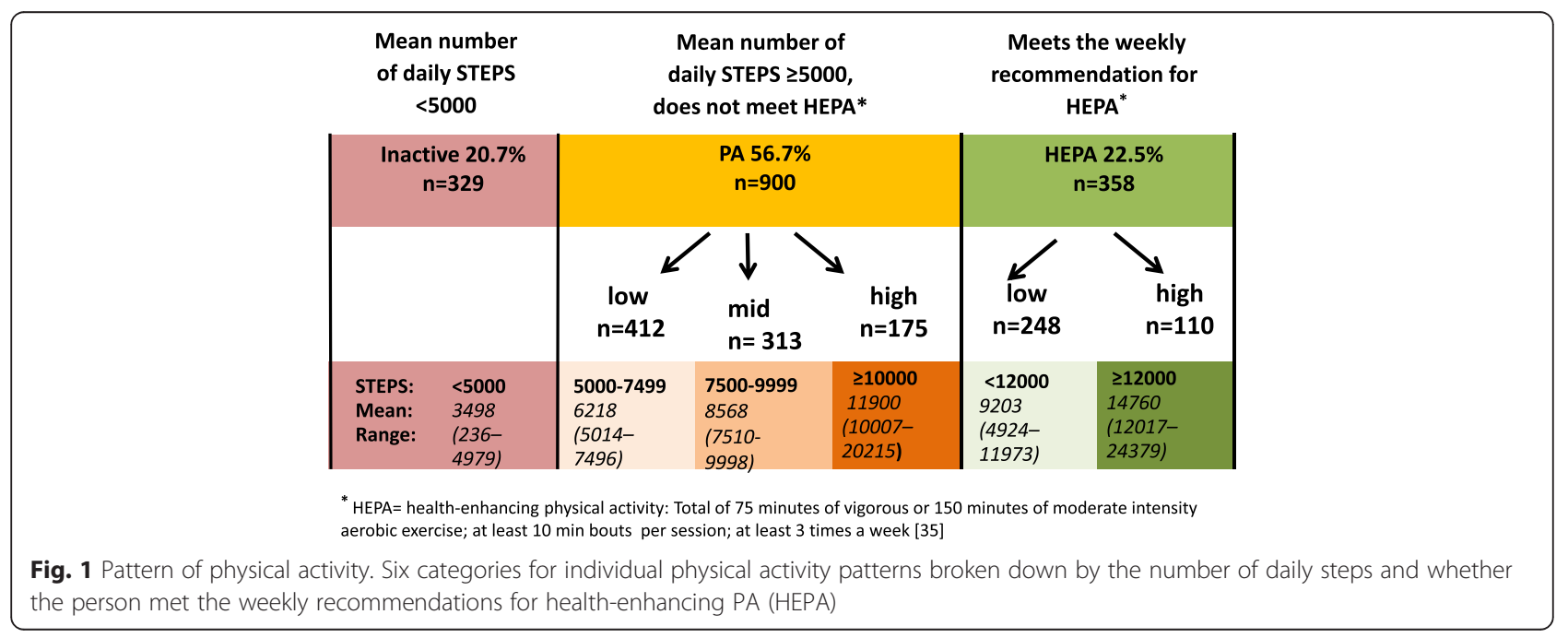


The results are presented in age and sex specific groups. Because of skewed distributions, gamma regression was used to test the difference between age groups and sex on SB- and MVPA-bouts. Sex comparisons were adjusted for age and age group comparisons for sex. For multiple comparisons Sidak-adjustment was used. $P$-value less than 0.05 was considered as statistically significant.

\section{Results}

The participants, who sufficiently wore the accelerometer for at least 4 days and at least during $10 \mathrm{~h}$ per day had on average higher education and better self-rated health and self-rated fitness than those who did not wear accelerometer or those who did not meet the criteria for sufficient wear time (Table 1). Most of the participants with sufficient accelerometer use had used it on six days during the 7-day-period. The mean waking wear time was $14 \mathrm{~h} 5$ min per day, men had 19 min longer mean waking wear time than women (Table 2). The mean age of the participants was 52 years (SD 14.7) and $57 \%$ of them were women. The mean weight was $84.9 \mathrm{~kg}$ (SD 14.6) for men and $70.7 \mathrm{~kg}$ (SD13.5) for women, and the mean height was $177.5 \mathrm{~cm}$ (SD 6.8) for men and $164.0 \mathrm{~cm}$ (SD 6.6) for women. The mean BMI was 26.9 (SD 4.2) for men and 26.3 (SD 4.8) for women. Thirtyeight percent of the participants had BMI $<25.0 \mathrm{~kg} / \mathrm{m}^{2}$, $38 \%$ had BMI between 25.0 and $29.9 \mathrm{~kg} / \mathrm{m}^{2}$ and $24 \%$ had $\geq 30 \mathrm{~kg} / \mathrm{m}^{2}$.

Irrespective of age and sex, the participants spent most of their waking hours sedentary. SB covered on average $59 \%$ and standing still $17 \%$ of the waking wear time. Participants spent $15 \%$ of their waking hours in light

Table 1 Background characteristics of the participants aged 18-85 years

\begin{tabular}{|c|c|c|c|c|c|}
\hline & & 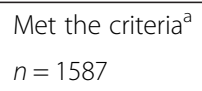 & $\begin{array}{l}\text { Did not meet the criteria } \\
n=263\end{array}$ & $\begin{array}{l}\text { Did not wear accelerometer } \\
n=190\end{array}$ & $p$-value ${ }^{b}$ \\
\hline \multirow[t]{2}{*}{ Sex } & Men, \% & 42.6 & 41.4 & 42.6 & 0.939 \\
\hline & Women, \% & 57.4 & 58.6 & 57.4 & \\
\hline \multirow[t]{6}{*}{ Age group } & $18-29, \%$ & 5.3 & 5.0 & 3.7 & 0.264 \\
\hline & $30-39, \%$ & 14.7 & 12.2 & 13.7 & \\
\hline & $40-49, \%$ & 22.9 & 22.8 & 22.1 & \\
\hline & $50-59, \%$ & 22.5 & 17.1 & 21.6 & \\
\hline & $60-69, \%$ & 20.4 & 24.7 & 26.3 & \\
\hline & $70-85, \%$ & 14.2 & 18.3 & 12.6 & \\
\hline \multirow[t]{4}{*}{ Education } & No vocational education, \% & 10.8 & 14.8 & 8.7 & 0.025 \\
\hline & Vocational education, \% & 62.1 & 66.4 & 66.8 & \\
\hline & University degree, \% & 27.1 & 18.8 & 24.5 & \\
\hline & Missing (n) & 87 & 13 & 6 & \\
\hline \multirow[t]{3}{*}{ Marital status } & Cohabited, \% & 76.2 & 67.6 & 81.0 & 0.003 \\
\hline & Single, \% & 23.8 & 32.4 & 19.0 & \\
\hline & Missing (n) & 87 & 13 & 6 & \\
\hline \multirow[t]{3}{*}{ Perceived health } & Good, \% & 47.7 & 38.8 & 42.9 & 0.022 \\
\hline & Fairly good/poor, \% & 52.3 & 61.2 & 57.1 & \\
\hline & Missing (n) & 88 & 13 & 6 & \\
\hline \multirow[t]{3}{*}{ Perceived fitness } & Good, \% & 40.8 & 30.2 & 33.7 & 0.002 \\
\hline & Fairly good/poor, \% & 59.2 & 69.8 & 66.3 & \\
\hline & Missing (n) & 94 & 15 & 6 & \\
\hline \multirow[t]{5}{*}{ HEPA } & Met the whole recommendation`, \% & 12.2 & 8.6 & 13.9 & 0.046 \\
\hline & Met aerobic part, \% & 28.3 & 22.0 & 28.9 & \\
\hline & Met musculoskeletal part, \% & 11.0 & 9.8 & 8.6 & \\
\hline & Did not meet the recommendation, \% & 48.6 & 59.6 & 48.7 & \\
\hline & Missing (n) & 27 & 8 & 3 & \\
\hline
\end{tabular}

at least 4 days, at least $10 \mathrm{~h} /$ day

${ }^{\mathrm{b}} \mathrm{Chi}{ }^{2}$-test

${ }^{C}$ met the whole recommendation: moderate intensity aerobic PA for at least 150 min per week, vigorous intensity PA for at least 75 min per week or an equal amount of MVPA spread throughout the week and musculoskeletal activity for at least twice a week [35] 
Table 2 The proportions of days (\%) with at least $10 \mathrm{~h}$ measurement-time and the mean accelerometer wearing time

\begin{tabular}{|c|c|c|c|c|c|c|c|}
\hline & & \multicolumn{6}{|l|}{ Age group } \\
\hline & & $18-29$ & $30-39$ & $40-49$ & $50-59$ & $60-69$ & $70-85$ \\
\hline & Men & $n=26$ & $n=101$ & $n=144$ & $n=162$ & $n=153$ & $n=90$ \\
\hline \multirow[t]{4}{*}{ Number of days meeting the $10 \mathrm{~h}$-criteria } & 4 & 3.8 & 13.9 & 13.2 & 13.0 & 10.5 & 14.4 \\
\hline & 5 & 15.4 & 19.8 & 20.8 & 21.0 & 15.7 & 23.3 \\
\hline & 6 & 61.5 & 45.5 & 45.8 & 47.5 & 54.2 & 53.3 \\
\hline & 7 & 19.2 & 20.8 & 20.1 & 18.5 & 19.6 & 8.9 \\
\hline \multirow[t]{3}{*}{ Wearing time } & Mean & 14 h 32 min & 14 h 32 min & $14 \mathrm{~h} 31 \mathrm{~min}$ & $14 \mathrm{~h} 21 \mathrm{~min}$ & 13 h 57 min & 13 h 39 min \\
\hline & SD & $57 \mathrm{~min}$ & 1 h 19 min & $1 \mathrm{~h} 20 \mathrm{~min}$ & $1 \mathrm{~h} 26 \mathrm{~min}$ & $1 \mathrm{~h} 19 \min$ & $1 \mathrm{~h} 31 \mathrm{~min}$ \\
\hline & Women & $n=58$ & $n=132$ & $n=219$ & $n=195$ & $n=171$ & $n=136$ \\
\hline \multirow[t]{4}{*}{ Number of days meeting the $10 \mathrm{~h}$-criteria } & 4 & 12.1 & 7.6 & 9.1 & 7.7 & 9.9 & 9.6 \\
\hline & 5 & 13.8 & 15.9 & 21.0 & 21.0 & 14.0 & 22.8 \\
\hline & 6 & 55.2 & 48.5 & 53.0 & 56.4 & 60.8 & 52.9 \\
\hline & 7 & 19.0 & 28.0 & 16.9 & 14.9 & 15.2 & 14.7 \\
\hline \multirow[t]{2}{*}{ Wearing time } & Mean & 13 h 43 min & 14 h 14 min & 14 h 16 min & 14 h 6 min & $13 \mathrm{~h} 38 \mathrm{~min}$ & $13 \mathrm{~h} 23 \mathrm{~min}$ \\
\hline & SD & 1 h 54 min & $1 \mathrm{~h} 2 \mathrm{~min}$ & $1 \mathrm{~h} 14 \mathrm{~min}$ & $1 \mathrm{~h} 8 \mathrm{~min}$ & $1 \mathrm{~h} 13 \mathrm{~min}$ & $1 \mathrm{~h} 14$ min \\
\hline
\end{tabular}

PA. The mean proportion of MVPA was on average $9 \%$ (moderate PA nearly $9 \%$, vigorous PA less than $1 \%$ ) (Fig. 2).

Figure 3a presents the mean daily hours of SB and standing still separately for the men and women. On average participants spent $8 \mathrm{~h} 20 \mathrm{~min}$ of their waking hours sedentary, in a sitting or reclining position and on average $2 \mathrm{~h} 20 \mathrm{~min}$ standing still. Men were on average more sedentary than women ( 8 h 40 min vs. 8 h 4 min, $p<0.001)$, while women were standing on average 27 min more per day than men $(p<0.001)$. When the breaks in SB were assessed as a mean number of standing-ups per day, the middle-aged participants broke the sedentary periods most often. The youngest group had on average 42 standing-ups per day, 30-39-year-olds had 51, 40-49-year-olds 48, 50-59-year-olds 47, 60-69year-olds 43 and those 70-years of age or older had 36 . Women had on average 3 more standing-ups per day than men $(p<0.05)$. Women had on average less long $(\geq 30 \mathrm{~min}$ ) sedentary bouts per day than the men $(2.6$ vs. 3.1, $p<0.001)$. Regarding the age-groups, the oldest ( $\geq 70$ years) and the youngest (18-29 years) participants had on average more long sedentary bouts per day than the other participants $(p<0.05)$. The participants aged 30-39 years had on average the smallest amount of long sedentary bouts (Fig. 3b).



Fig. 2 Proportions of sedentary behavior, standing still and physical activity. Proportions of sedentary behavior, standing still, and light, moderate and vigorous physical activity in relation to measurement time by age-group and sex 

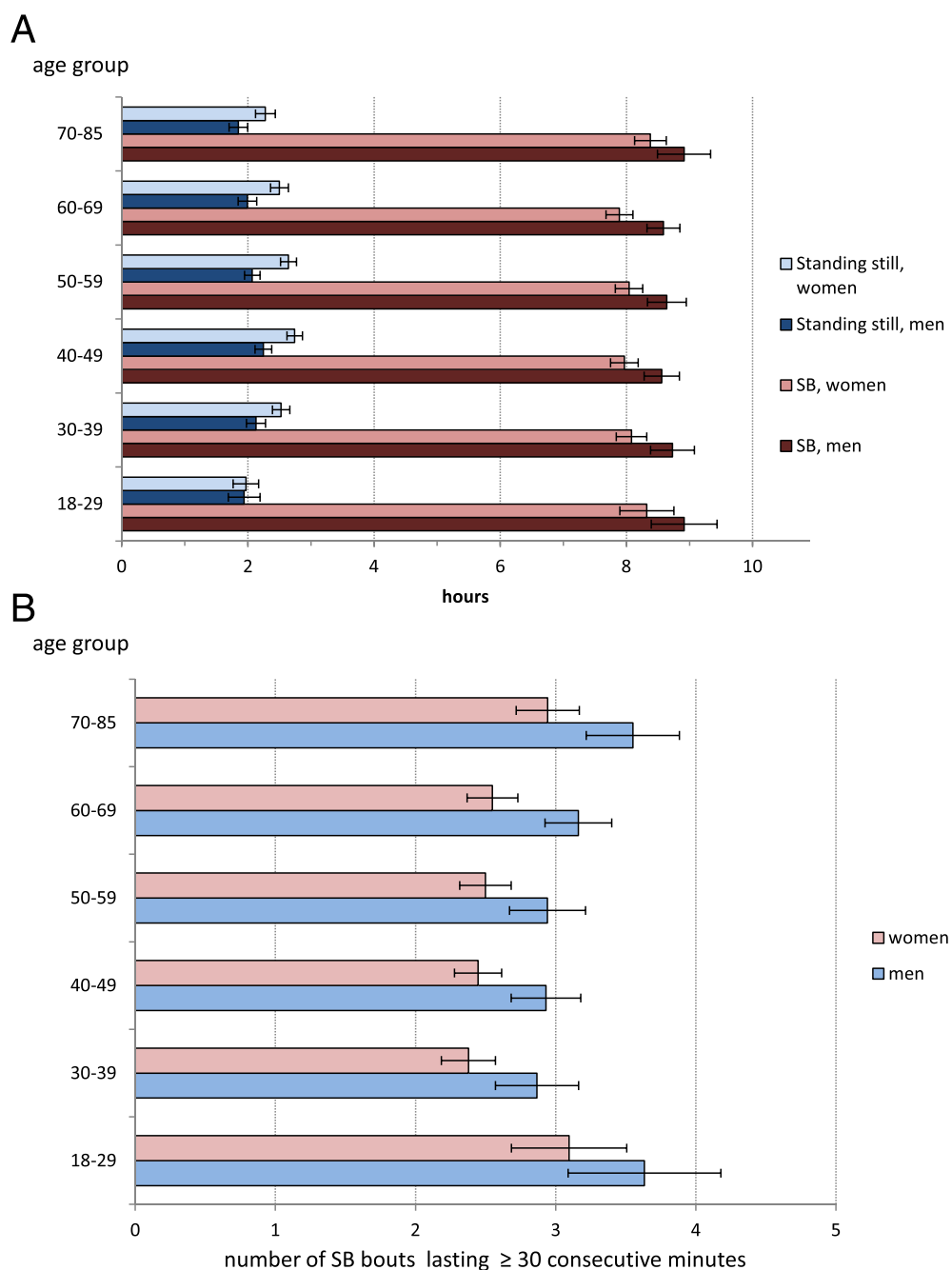

Fig. 3 a Mean daily hours of sedentary behavior and standing still. Mean daily hours of sedentary behavior and standing still with $95 \%$ confidence interval by age-group and sex. $\mathbf{b}$ Mean number of sedentary bouts lasting at least 30 consecutive minutes. Mean number of sedentary bouts lasting at least 30 consecutive minutes with $95 \%$ confidence interval by sex

Figure 4a presents the daily hours of light PA and MVPA. The mean time of light PA was slightly over $2 \mathrm{~h}$ (2 $\mathrm{h} 8 \mathrm{~min})$ and that for MVPA $1 \mathrm{~h} 17 \mathrm{~min}$ per day. The 30-60-year-olds had on average more light PA than the youngest and the oldest participants, but the individual variation was large. In each age- and sex-group there were both very active and very inactive participants. The youngest participants had more MVPA than the older groups, but again individual variation was large, especially among the men aged under 30 years. Participants younger than 30 years took on average 9043 steps per day (95\% CI 8351-9736), while the participants aged over 70 years took on average 5183 steps (95\% CI 4762-5604). Figure 4b and 4c presents the mean number of MVPA bouts lasting 5-15 consecutive minutes (4b) and the number of bouts exceeding 15 consecutive minutes (4c). There were no statistically significant sex differences in these bouts. The oldest participants ( $\geq 70$ years) had on average less MVPA bouts of 5-15 min than all the younger participants $(p<0.001$ to $p=0.035$ ). Regarding the longer bouts, the oldest participants differed only from the 60-69-year-olds $(p=0.005)$ and 40-49-year-olds $(p=0.020)$.

Regarding the proposed classification scheme of PApatterns (see Fig. 1), over a half of the participants (57 \%) belonged to PA-group, not meeting the HEPA recommendation, but taking on average over 5000 steps per day. Men and women were equally distributed into each group, but the mean age of the inactive-group was higher than that of the other groups $(p<0.001)$.

Figure $5 \mathrm{a}$ and $\mathrm{b}$ present the age- and sex-adjusted SB, standing and PA times in the six PA categories. Highest average amount of sedentary time was recorded in the inactive-group (Fig. 5a) which also had higher number 

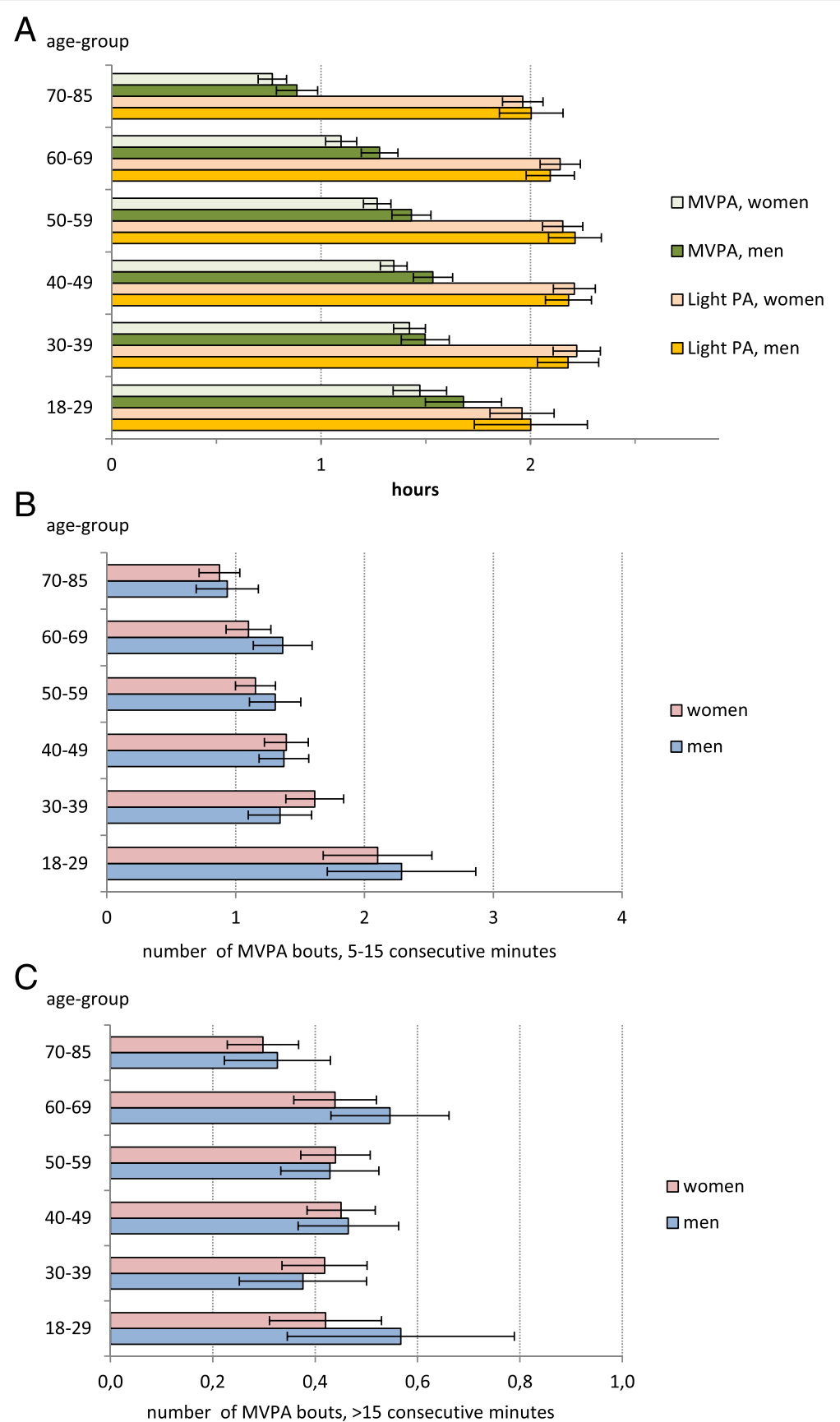

Fig. 4 a Mean daily hours of physical activity. Mean daily hours of light and moderate-to-vigorous physical activity with $95 \%$ confidence interval by age-group and sex. $\mathbf{b}$ Mean number of moderate-to-vigorous physical activity bouts of 5-15 consecutive minutes. Mean number of moderate-to-vigorous physical activity bouts lasting 5-15 consecutive minutes with $95 \%$ confidence interval by sex. c Mean number of moderate-to-vigorous physical activity bouts over 15 consecutive minutes. Mean number of moderate-to-vigorous physical activity bouts lasting over 15 consecutive minutes with $95 \%$ confidence interval by sex

of long ( $\geq 30 \mathrm{~min})$ sedentary bouts than the other groups $(p<0.001)$. PA-high and HEPA-high -groups had on average similar amount of SB (7 h 20 min), while HEPA-low group was on average more sedentary (8 h $30 \mathrm{~min})$.

The HEPA-high -group spent on average more time in MVPA than the other groups (Fig. 5b). The HEPA-low- group, on the other hand, had on average less MVPA than the PA-high-group $(p<0.001)$, less light activity than all the PA-groups $(p<0.01)$ and less standing time than the PA-mid and-high-groups $(p<0.001)$. PA-high-group had on average the greatest amount of light PA and the least number of long ( $\geq 30 \mathrm{~min})$ sedentary bouts, 


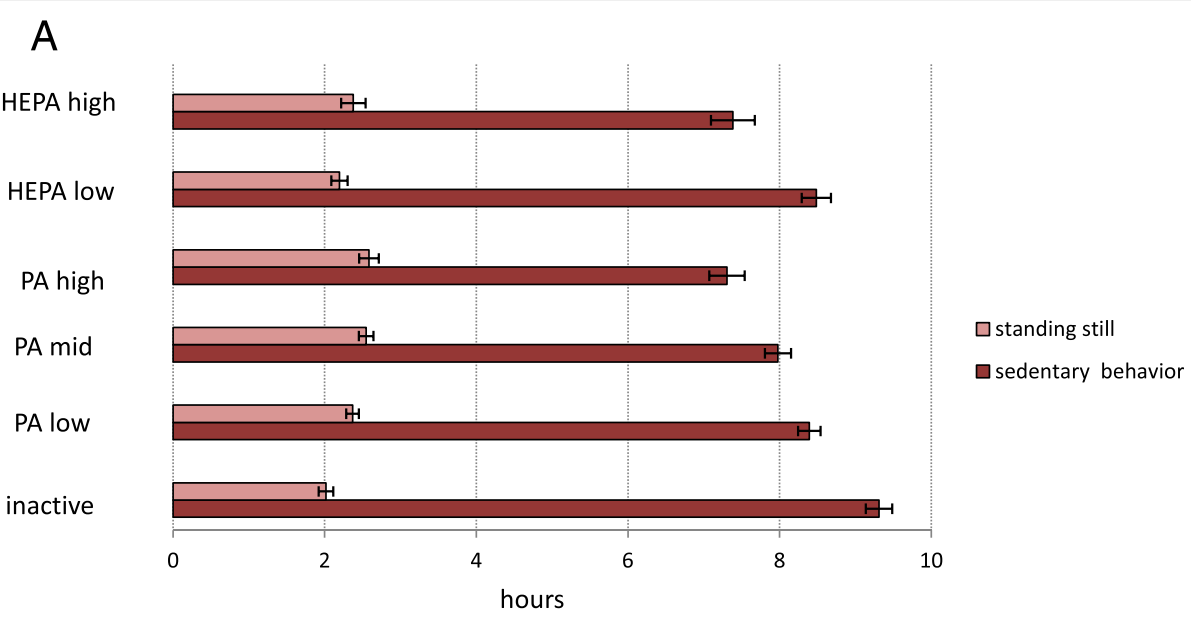

B

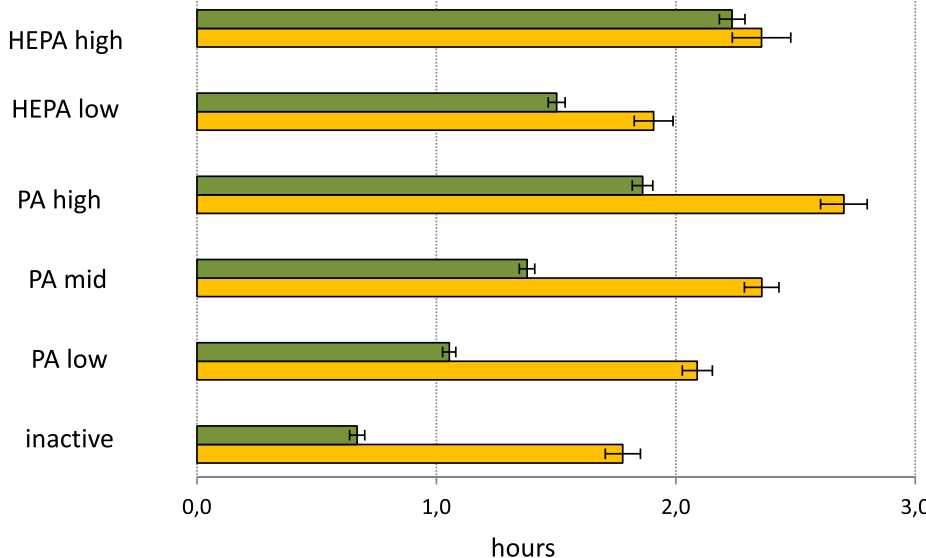

$\square$ MVPA

$\square$ light PA

Fig. 5 a Mean hours of sedentary behavior and standing still according to physical activity categorization. Mean hours of sedentary behavior and standing still in six categories of physical activity categorization with $95 \%$ confidence interval, adjusted for age and sex. b Mean hours of physical activity according to physical activity categorization. Mean hours of light and moderate-to-vigorous physical activity in six PA-categories with $95 \%$ confidence interval, adjusted for age and sex

although the difference in the number of long sedentary bouts between HEPA-high and PA-high-groups was not statistically significant.

\section{Discussion}

The present study measured objectively various features of SB and PA using raw tri-axial accelerometer data. According to the results participants spent on average $59 \%$ of their waking hours sedentary and additional $17 \%$ by standing still. Light activity covered $15 \%$ and MVPA less than one tenth of the waking wear time. The proposed classification scheme for PA was generally able to detect logical differences between the accelerometermeasured features of SB and PA in the six proposed PA categories. In the future studies as well as in the practise of PA counselling the novel classification may help in identifying individual activity patterns and thus facilitate to target actions to reduce $\mathrm{SB}$ and promote $\mathrm{PA}$ more precisely in a personalized fashion. Consideration of steps in the PA categorization revealed clear differences between and within the traditionally used categories (inactive, active, HEPA).

Despite weaknesses in the criterion validity of previously reported count-based measurements [23], the average $59 \%$ daily sedentary time in the present study sample is in line with previously reported values (55-62\%) [24-27, 29]. In terms of hours the proportion of SB corresponds on average to $7.3-9 \mathrm{~h}$ per day $[24-27,29]$. The mean SB (8.3 h per day) identified in the present study is also well in line with the former objective findings, as is the finding that men spent, on average, more time sedentary than women [27, 41].

Recent studies have reported that short breaks in SB are beneficial for health $[42,43]$ and may be particularly important for cardio-metabolic health [7]. It has also been suggested that breaking up sedentary time regularly 
and replacing it by light intensity PA is more amenable to change than increasing the amount of MVPA [2]. In the present study people aged 30 to 60-years had on average more breaks in SB than the youngest and oldest age groups. Interestingly, and according to the aforementioned hypothesis, they also had the greatest time of light PA.

In the present study, the mean daily proportion of light PA was $15 \%$ which is considerably less than the $39 \%$ reported by Healy et al. [26] and Spittaels et al. [24] and the $34 \%$ of low-intensity PA and lifestyle activity reported by Hansen et al. [27]. This is most likely due to differences in the representativeness of the study populations as well as differences in measurement methods and analysis algorithms. Most of the previous population studies have not differentiated standing from SB. However, it is well-agreed that standing is a separate behavior from SB [3, 44]. Some standing with slight movement or swaying might have been classified as light PA in the previous studies. The rationale to separate standing from light PA in the present study was that the energy expenditure of standing is lower than that of light PA [45], while despite the static nature of standing, as a prolonged continuous behavior it may lead to specific health problems [46].

The proportion of MVPA in the present study sample was on average $9 \%$ of waking hours. The values are somewhat higher than those reported for adults in previous studies, from $4 \%[26,27,29]$ to $6 \%$ [24]. The comparison between studies is challenging since the analysis algorithms [21] as well as the cut-points for MVPA [14] have varied a lot between the studies.

The proposed classification scheme for individual PA-patterns was able to detect logical differences in parameters describing SB and PA between the groups. The inactive-group had the greatest amount of SB. Although the PA-high group did not meet the HEPA recommendation [35], the mean total time of MVPA in that group was greater than that in the HEPA-low -group. PA-high -group had also the greatest amount of light PA. For public health reasons, on a population level, it is important to promote total PA and to decrease SB. These two targets need different tools and actions and the proposed PA-patterns may help to target these actions appropriately. For example the proposed PA-pattern indicates that being active enough to meet HEPA recommendation, as in the HEPA-low-group, may simultaneously mean that the total amount of PA is lower and the amount of SB is higher than in the PA-high group not meeting the recommendation. Thus, replacing SB by light PA might be the most important target in HEPA-low group. In the PA-high -group, in turn, promotion of longer MVPA-bouts to meet the HEPA recommendation could be a way to increase health benefits of PA while keeping the previous level of light activity. The inactive group needs more PA at any intensity while the amount of SB needs to be reduced. The most feasible way of doing that could be adding active breaks into sedentary time and promoting light PA in terms of active commuting and everyday activities. Thus, the proposed categorization offers a more specific and more personalized tool to identify PA-patterns than the traditionally used categorization based on HEPA-criteria only.

\section{Strengths and limitations}

The strength of the present study is that several features of PA and SB were measured with a tri-axial accelerometer collecting data in raw mode as recommended [21, 31]. The data was analyzed with novel, universal and valid algorithms [30, 32] and the study sample included both men and women within a wide age range. Although the accelerometer used in the present study is not widely used, the analysis methods are universal and can be used with any tri-axial accelerometer collecting data in raw mode [30]. Weaknesses of the study were that the currently used analysis algorithms may not accurately recognize movements performed only with lower-or upper-extremities (eg. gym exercises) and movements performed in supine position (eg. pilates). Also the intensities of cycling and Nordic skiing are not adequately captured at the moment [22] Further, accelerometer used in the study was not waterresistant which means that water-activities, like swimming, were not included. The cross-sectional design of the study is also a limitation to causal interpretation of the results and trend analysis. The Health 2011 nonparticipation and representativeness has been presented in more detail elsewhere [47]: in general, the participation to Health 2011 sample was acceptable 72,5 \% but lower participation was observed among young age-groups, men and among those with low educational attainment. Moreover, the overall participation rate of $50 \%$ from the original sample, limits the generalization of the results to the adult Finnish population. Also, unequal participation of the age- and sex groups limits the generalizability of the results.

\section{Conclusions}

Finnish adults in this study were sedentary almost $60 \%$ of the waking hours, and the majority of daily PA was light. From a public health perspective it is important to find effective ways to decrease SB as well as to increase the level of daily PA. A proposed classification scheme based on the validated analysis of the tri-axial acceleration data and number of daily steps may help in personalized targeting and planning PA promotion policies appropriately. 
In the future it is important to assess objectively measured SB and PA in representative study populations in order to get representative and reliable data on the level of these behaviors in the population. Furthermore, the dose-response relationships between objectively measured PA and SB and various indicators of health and well-being, for example lipid- and glucose metabolism [42], coronary heart diseases [48] and low back pain [49], need to be studied. Different PA and SB bouts over day need to be studied in more detail.

Especially the health effects of objectively measured light, sporadic activity and on the other hand effects of different bouts of PA and SB and breaks in SB on health outcomes need to be elaborated.

\section{Acknowledgements}

The authors would like to acknowledge the contribution of the Department of Health, Functional Capacity and Wellbeing at the National Institute for Health and Welfare for collecting and preparing the Health 2011 data.

\section{Funding}

The study was financially supported by the Finnish Ministry of Education and Culture (data collection and analysis). Tomi Mäki-Opas was supported by the Academy of Finland (\#266759).

\section{Availability of data and materials}

The Health 2011 Study data is maintained by the National Institute for Health and Welfare (THL). The data is available for research purposes from the www.terveys2011.info/aineisto after the research proposal has been accepted by the executive scientific committee of THL. The information on the interviews, questionnaires and health examination can also be found in English from the web page.

\section{Authors' contributions}

$\mathrm{PH}$ participated in the design of the study, analyzed the data, interpreted the results and was responsible for preparing the manuscript. JS participated in the design of the study, interpreted the results and revised the manuscript. $\mathrm{HV}-\mathrm{Y}$ was responsible for processing the accelerometer data, interpreted the results and revised the manuscript. HS participated in the design of the study, interpreted the results and revised the manuscript. KT analyzed the data and revised the manuscript. HV participated in the design of the study, interpreted the results and revised the manuscript. TM-O participated in the design of the study, interpreted the results and revised the manuscript. TV was responsible for the study design, interpreted the results and revised the manuscript. All authors read and approved the final manuscript.

\section{Competing interests}

The authors declare that they have no competing interests.

\section{Consent for publication}

Not applicable.

\section{Ethics approval and consent to participate}

The Coordinating ethics committee of the Hospital District of Helsinki and Uusimaa gave an ethical approval for the study (45113/03100/11). All participants gave a signed informed consent before participation.

\section{Author details}

${ }^{1}$ The UKK Institute for Health Promotion Research, Tampere, Finland. ${ }^{2}$ Department of Welfare, The National Institute for Health and Welfare (THL), Helsinki, Finland. ${ }^{3}$ Department of Health, The National Institute for Health and Welfare (THL), Helsinki, Finland.

Received: 11 March 2016 Accepted: 25 August 2016

Published online: 01 September 2016

\section{References}

1. Lee IM, Shiroma EJ, Lobelo F, Puska P, Blair SN, Katzmarzyk PT. Effect of physical inactivity on major non-communicable diseases worldwide: an analysis of burden of disease and life expectancy. Lancet. 2012;380:219-29.

2. Dempsey PC, Owen N, Biddle SJH, Dunstan DW. Managing sedentary behavior to reduce the risk of diabetes and cardiovascular disease. Curr Diab Reb. 2014;14:522

3. Sedentary Behavior Research Network. Letter to the editor: Standardized use of the terms "sedentary" and "sedentary behaviors". Appl Physiol Nutr Metab. 2012;37:540-2.

4. Chastin SF, Schwarz U, Skelton DA. Development of a consensus taxonomy of sedentary behaviors (SIT): report of Delphi Round 1. PLoS One. 2013;8:e82313.

5. Thorp AA, Owen N, Neuhaus M, Dunstan DW. Sedentary behaviors and subsequent health outcomes in adults a systematic review of longitudinal studies, 1996-2011. Am J Prev Med. 2011;41:207-15.

6. de Rezende LFM, Lopes MR, Rey-Lopez JP, Matsudo VKR, Luiz OC. Sedentary behavior and health outcomes: an overview of systematic reviews. PloS One. 2014;9:e105620.

7. Carson V, Wong SL, Winkler E, Healy GN, Colley RC, Tremblay MS. Patterns of sedentary time and cardiometabolic risk among Canadian adults. Prev Med. 2014;65C:23-7.

8. Borodulin K, Karki A, Laatikainen T, Peltonen M, Luoto R. Daily sedentary time and risk of cardiovascular disease: The National FINRISK 2002 Study. J Phys Act Health. 2015;12:904-8.

9. Mork PJ, Westgaard RH. Back posture and low back muscle activity in female computer workers: a field study. Clin Biomech (Bristol, Avon). 2009;24:169-75.

10. Zhai L, Zhang $Y$, Zhang D. Sedentary behaviour and the risk of depression: a meta-analysis. BJSM Online first. doi: 10.1136/bjsports-2014-093613.

11. Koster A, Caserotti P, Patel KV, Matthews CE, Berrigan D, Van Domelen DR, et al. Association of sedentary time with mortality independent of moderate to vigorous physical activity. PLoS One. 2012;7:e37696.

12. Biswas A, Oh Pl, Faulkner GE, Bajaj RR, Silver MA, Mitchell MS, et al. Sedentary time and its association with risk for disease incidence, mortality and hospitalization in adults. Ann Intern Med. 2015;162:123-31.

13. Bakrania K, Edwardson CL, Bodicoat DH, Esliger DW, Gill JM, Kazi A, et al. Associations of mutually exclusive categories of physical activity and sedentary time with markers of cardiometabolic health in English adults: a cross-sectional analysis of the Health Survey for England. BMC Public Health. 2016;16(1):25. Epub 2016/01/13

14. Gorman E, Hanson HM, Yang PH, Khan KM, Liu-Ambrose T, Ashe MC. Accelerometry analysis of physical activity and sedentary behavior in older adults: a systematic review and data analysis. Eur Rev Aging Phys Act. 2014;11:35-49.

15. Haskell WL. Physical activity by self-report: a brief history and future issues. J Phys Act Health. 2012;9(S1):5-10

16. Clark BK, Healy GN, Winkler EA, Gardiner PA, Sugiyama T, Dunstan DW, et al. Relationship of television time with accelerometer-derived sedentary time: NHANES. Med Sci Sports Exerc. 2011;43:822-8.

17. Helmerhorst HJ, Brage S, Warren J, Besson H, Ekelund U. A systematic review of reliability and objective criterion-related validity of physical activity questionnaires. Int J Behav Nutr Phys Act. 2012:9:103.

18. Craig CL, Marshall AL, Sjostrom M, Bauman AE, Booth ML, Ainsworth BE, et al. International physical activity questionnaire: 12-country reliability and validity. Med Sci Sports Exerc. 2003;35:1381-95.

19. Matthews CE, Moore SC, George SM, Sampson J, Bowles HR. Improving selfreports of active and sedentary behaviors in large epidemiologic studies. Exerc Sport Sci Rev. 2012;40:118-26.

20. Janssen X, Cliff DP. Issues related to measuring and interpreting objectively measured sedentary behavior data. Meas Phys Educ Exerc Sci. 2015:19:116-24

21. Matthews CE, Hagströmer M, Pober DM, Bowles HR. Best practices for using physical activity monitors in population-based research. Med Sci Sports Exerc. 2012;44(S1):S68-76.

22. Strath SJ, Kaminsky LA, Ainsworth BE, Ekelund U, Freedson PS, Gary RA, et al Guide to the assessment of physical activity: Clinical and research applications: a scientific statement from the American Heart Association. Circulation. 2013;128:2259-79.

23. Kim Y, Welk GJ. Criterion validity of competing accelerometry-based activity monitoring devices. Med Sci Sports Exerc. 2015;11:2456-63. 
24. Spittaels H, Van Cauwenberghe E, Verbestel V, De Meester F, Van Dyck D, Verloigne $M$, et al. Objectively measured sedentary time and physical activity time across the lifespan: a cross-sectional study in four age groups. Int J Behav Nutr Phys Act. 2012;9:149.

25. Matthews CE, Chen KY, Freedson PS, Buchowski MS, Beech BM, Pate RR, et al. Amount of time spent in sedentary behaviors in the United States, 2003-2004. Am J Epidemiol. 2008;167:875-81.

26. Healy GN, Wijndaele K, Dunstan DW, Shaw JE, Salmon J, Zimmet PZ, et al. Objectively measured sedentary time, physical activity, and metabolic risk: the Australian Diabetes, Obesity and Lifestyle Study (AusDiab). Diabetes Care. 2008:31:369-71.

27. Hansen BH, Kolle E, Dyrstad SM, Holme I, Anderssen SA. Accelerometerdetermined physical activity in adults and older people. Med Sci Sports Exerc. 2012:44:266-72.

28. Schuna Jr JM, Johnson WD, Tudor-Locke C. Adult self-reported and objectively monitored physical activity and sedentary behaviour: NHANES 2005-2006. Int J Behav Nutr Phys Act. 2013;10:126.

29. Hagstromer M, Oja P, Sjostrom M. Physical activity and inactivity in an adult population assessed by accelerometry. Med Sci Sports Exerc. 2007;39:1502-8.

30. Vähä-Ypyä H, Vasankari T, Husu P, Suni J, Sievänen H. A universal, accurate intensity-based classification of different physical activities using raw data of accelerometer. Clin Physiol Funct Imaging. 2015;35:64-70.

31. Westerterp KR. Reliable assessment of physical activity in disease: an update on activity monitors. Curr Opin Clin Nutr Metab Care. 2014;17:401-6.

32. Vähä-Ypyä H, Vasankari T, Husu P, Mänttäri A, Vuorimaa T, Suni J, et al. Validation of cut-points for evaluating the intensity of physical activity with accelerometry-based mean amplitude deviation (MAD). Plos One. 2015;10:e0134813.

33. Koskinen S, Lundqvist A, Ristiluoma N, editors. Health, functional capacity and welfare in Finland in 2011. Helsinki: National Institute for Health and Welfare (THL), Report 68/2012; 2012.

34. Celis-Morales CA, Perez-Bravo F, Ibanez L, Salas C, Bailey ME, Gill JM. Objective vs. self-reported physical activity and sedentary time: effects of measurement method on relationships with risk biomarkers. PloS One. 2012; 7(5):e36345

35. 2008 Physical activity guidelines for Americans: Be active, healthy and happy! Rockville: U.S. Department of Health and Human Services; 2008.

36. Ying $H$, Silex $C$, Schnitzer A, Leonhardt S, Schiek M. Automatic step detection in the accelerometer signal. In: Proceedings of the 4 th International workshop on wearable and implantable body sensor networks (BSN 2007) IFMBE Proceedings, vol. 13. 2007. p. 80-5.

37. Mizell D. Using gravity to estimate accelerometer orientation. In: Proceedings seventh IEEE international symposium on wearable computers, vol. 2005. 2003. p. 252-3.

38. Sievänen H, Vähä-Ypyä H, Husu P, Suni J, Vasankari T. A universal method for accurate classification of physical activity and sedentary behavior with tri-axial accelerometry. Med Sci Sports Exerc. 2014;46:S438.

39. Tudor-Locke C, Craig CL, Brown WJ, Clemes SA, De Cocker K, Giles-Corti B, et al. How many steps/day are enough? For adults. Int J Behav Nutr Phys Act. 2011;8:79.

40. Tudor-Locke C, Craig CL, Thyfault JP, Spence JC. A step-defined sedentary lifestyle index: <5000 steps/day. Appl Physiol Nutr Metab. 2013;38:100-14.

41. Stamatakis $E$, Hamer M, Tilling K, Lawlor DA. Sedentary time in relation to cardio-metabolic risk factors: differential associations for self-report vs accelerometry in working age adults. Int J Epidemiol. 2012;41:1328-37.

42. Duvivier BM, Schaper NC, Bremers MA, van Crombrugge G, Menheere PP, Kars $\mathrm{M}$, et al. Minimal intensity physical activity (standing and walking) of longer duration improves insulin action and plasma lipids more than shorter periods of moderate to vigorous exercise (cycling) in sedentary subjects when energy expenditure is comparable. PLoS One. 2013;8:e55542.

43. Thosar SS, Bielko SL, Mather KJ, Johnston JD, Wallace JP. Effect of prolonged sitting and breaks in sitting time on endothelial function. Med Sci Sports Exerc. 2015;47:843-9.

44. Katzmarzyk PT. Standing and mortality in a prospective cohort of Canadian adults. Med Sci Sports Exerc. 2014;46:940-6.

45. Tudor-Locke C, Schuna Jr JM, Frensham LJ, Proenca M. Changing the way we work: elevating energy expenditure with workstation alternatives. Int J Obes (Lond). 2014;38:755-65.

46. Halim I, Omar AR, Saman AM, Othman I. Assessment of muscle fatigue associated with prolonged standing in the workplace. Saf Health Work. 2012;3:31-42.
47. Härkänen T, Karvanen J, Tolonen H, Lehtonen R, Djerf K, Juntunen T, Koskinen S. Systematic handling of missing data in complex study designsexperiences from the Health 2000 and 2011 Surveys. J Appl Stat. 2016. doi:10.1080/02664763.2016.1144725. Published online 18 Feb 2016.

48. Ahmed HM, Blaha MJ, Nasir K, Rivera JJ, Blumenthal RS. Effects of physical activity on cardiovascular disease. Am J Cardiol. 2012;109:288-95.

49. Heneweer H, Picavet HS, Staes F, Kiers H, Vanhees L. Physical fitness, rather than self-reported physical activities, is more strongly associated with low back pain: evidence from a working population. Eur Spine J. 2012;21:1265-72.

\section{Submit your next manuscript to BioMed Central and we will help you at every step:}

- We accept pre-submission inquiries

- Our selector tool helps you to find the most relevant journal

- We provide round the clock customer support

- Convenient online submission

- Thorough peer review

- Inclusion in PubMed and all major indexing services

- Maximum visibility for your research

Submit your manuscript at www.biomedcentral.com/submit
Ciomed Central 